\title{
Complete Genome Sequence of Aconitum Amalgavirus 1, a Distinct Member of the Genus Amalgavirus
}

Fan Li ( $\square$ fanli@ynau.edu.cn )

Yunnan Agricultural University https://orcid.org/0000-0002-4991-9419

Jie Yang

Yunnan Agricultural University

Ping-Xiu Lan

Yunnan Agricultural University

Jin-Ming Li

Yunnan Agricultural University

Xiao-Jiao Chen

Yunnan Agricultural University

Guan-Lin Tan

Yunnan Agricultural University

Tai-Yun Wei

Fujian Agriculture and Forestry University

Ru-Hui Li

USDA-ARS,National Germplasm Resources Laboratory

\section{Research Article}

Keywords:

Posted Date: January 27th, 2022

DOI: https://doi.org/10.21203/rs.3.rs-1286824/v1

License: (1) (1) This work is licensed under a Creative Commons Attribution 4.0 International License. Read Full License 
Complete genome sequence of Aconitum amalgavirus 1, a distinct member of the genus Amalgavirus

Jie Yang ${ }^{1 \S}$, Ping-Xiu Lan ${ }^{1 \S}$, Jin-Ming Li ${ }^{1}$, Xiao-Jiao Chen ${ }^{1}$, Guan-Lin Tan ${ }^{2}$, Tai-Yun Wei ${ }^{3}$, Ru-Hui Li ${ }^{4 *}$, Fan $\mathrm{Li}^{1 *}$

${ }^{1}$ State Key Laboratory for Conservation and Utilization of Bio-Resources in Yunnan, Yunnan Agricultural University, Kunming 650201, China

${ }^{2}$ Modern Education Technology Center, Yunnan Agricultural University, Kunming 650201, China

${ }^{3}$ State Key Laboratory of Ecological Pest Control for Fujian and Taiwan Crops, Fujian Agriculture and Forestry University, Fuzhou 350002, China

${ }^{4}$ USDA-ARS, National Germplasm Resources Laboratory, Beltsville, MD 20705, USA

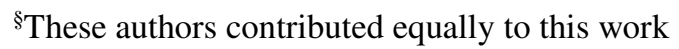

*Corresponding authors

Ruhui Li: Ruhui.Li@ars.usda.gov

Fan Li: fanli@ynau.edu.cn

\begin{abstract}
A novel virus named Aconitum amalgavirus 1 (AcoAV-1) was identified from Chinese aconite (Aconitum carmichaelii) plants with viral disease symptoms. The genome of this monopartite dsRNA virus is 3,370 nucleotides long, which containing two partially overlapping open reading frames encoding a putative coat protein and RNA dependent RNA polymerase, respectively. AcoAV-1 shares 34.9-50.7\% amino acid sequence identities to other amalgaviruses. Phylogenetic analyses group AcoAV-1 with blueberry latent virus and other related viruses in a clade, suggesting that it belongs to the genus Amalgavirus in the family Amalgaviridae.
\end{abstract}

\title{
Introduction
}

Chinese aconite (Aconitum carmichaelii), also known as Fuzi or Chuanwu, is a native species of the family Ranunculaceae in East Asia and has been used in traditional Chinese medicine for anti-inflammation and anti-arrhythmia purpose for thousand years. This herb has been cultivated in a large scale as medicinal plant since ancient time in China and is also cultivating as medicinal and/or 
ornamental plant in other countries [1]. Its tuberous roots are commonly applied for various diseases such as rheumatic fever, painful joints and some endocrinal disorders since they are rich in alkaloids[2]. Up to date, there are three viruses have been reported to infect Aconitum spp., which are Aconitum latent virus(AcLV) [3], cucumber mosaic virus(CMV) [4], and Aconite virus A (AcVA) [5]. Among them, AcVA is probably the only virus infect A. carmichaelii.

Amalgaviridae is a recently recognized family of double-stranded RNA (dsRNA) viruses that contains two genera, Zybavirus and Amalgavirus. Genus Zybavirus so far comprises only viruses isolated from fungal hosts and only one species of Zygosaccharomyces bailii virus $Z$ was assigned by ICTV. Genus Amalgavirus includes nine species of Allium cepa amalgavirus 1, Allium cepa amalgavirus 2, Blueberry latent virus, Rhododendron virus A, Southern tomato virus, Spinach amalgavirus 1, Vicia cryptic virus $M$, Zoostera marina amalgavirus 1, and Zoostera marina amalgavirus 2, which all infecting plants (https://talk.ictvonline.org/taxonomy/). Among the nine amalgaviruses, southern tomato virus (STV), vicia cryptic virus M (VCV-M), Zoostera marina amalgavirus 1 (ZmAV1), and Zoostera marina amalgavirus 2 (ZmAV2) have been reported in China [6-8]. In addition, more than fifteen other amalgaviruses had been submitted to NCBI but still unassigned by ICTV[9].

Amalgavirus is a group of monopartite dsRNA viruses, their genomes of approximately $3.5 \mathrm{~kb}$ in length contain two partially overlapping open reading frames (ORFs). ORF1 encodes a putative coat protein (CP), and ORF2 encodes a RNA-dependent RNA polymerase (RdRp) [10]. In addition, the fusion protein ORF1+2 is predicted to be expressed via a +1 programmed ribosomal frameshifting (PRF) event during the translation of ORF1 [9]. The +1 PRF motif sequence UUU_CGN (underline indicates the codon boundary for ORF1; N, any nucleotide), is prevalent in most amalgaviruses [8, 9]. When it occurs, the $+1 \mathrm{PRF}$, the codon frame changes from UUU_ CGN (ORF1) to U_UUC_GNN (ORF2). Members of the genus Amalgavirus are transmitted vertically via seeds, and are not thought to be capable of efficient extracellular transmission, unless mediated by an unknown vector[11].

\section{Identification of a novel aconitum amalgavirus}

In 2017, symptoms of foliar mosaic, mottle, necrosis and chlorosis were observed on Chinese aconite plants in a field in Zhanyi County, Yunnan Province, China. Symptomatic leaves were collected from each of seventeen diseased plants. A pooled sample of five aconite plants was subjected to 
high-throughput sequencing (HTS) using an Illumina HiSeq X-ten platform (BioMarker Biotech Co., Ltd, Beijing, China). The RNA reads of 150 bp were analyzed using CLC Genomics Workbench 15 (QIAGEN, Germantown, USA). After trimming, a total of 13,635,451 paired-end reads were obtained, and 14,423 contigs [>200 nucleotides (nt)] were de novo generated for BLASTx search. A large contig of 3,282 nt was identified to share the highest sequence identity (54\%) with blueberry latent virus (BBLV, GenBank accession number NC_014593) at the fusion protein, suggesting that it belongs to a distinct amalgavirus.

To verify the presence of the novel amalgavirus in the field aconite plant samples, total RNA was extracted from each of the five samples used for HTS using EasyPure ${ }^{\circledR}$ Plant RNA Kit (TransGen Biotech, Beijing, China). Four pairs of overlapping primers were designed according to the contig sequence (Table 1) and used for RT-PCR with PrimeScript ${ }^{\mathrm{TM}}$ One-Step RT-PCR Kit Ver. 2 (TaKaRa Biotechnology Co. Ltd., Dalian, China). PCR products were cloned into pMD19-T vector (TaKaRa Biotechnology Co. Ltd., Dalian, China) and sequenced (BGI, Guangzhou, China). The 5'-end and 3'-end sequences of the virus were obtained according to the method described by Lan et al[12]. The genome sequence was assembled and analyzed by SeqMan in the Lasergene v7.1 package (DNASTAR Inc., Madison, WI, USA). Phylogenetic analysis was performed in MEGA7 using 1000 bootstrap replicates by Neighbor-Joining method.

\section{Genomic properties of the novel amalgavirus}

The complete genomic RNA sequence of the virus was determined to be $3,370 \mathrm{nt}$ in its positive strand, including the 5'-untranslated region (UTR) of $160 \mathrm{nt}$ and 3'-UTR of $58 \mathrm{nt}$ (GenBank acc. no. MZ389237). Sequence analysis reveals that the virus has a genomic structure that is typical of amalgaviruses (Fig. 1a). ORF1 encodes a putative coat protein of 367 amino acids (aa) with a molecular mass of $40.6 \mathrm{kDa}$. The second protein is predicted to be an ORF1+2 fusion protein of 1,049 aa in length with a molecular mass of $118.7 \mathrm{kDa}$. The fusion protein is probably involved in mediation of virus replication. The putative +1 PRF motif sequence is predicted to be ${ }^{989}{ }^{9 U U}$ _CGA ${ }^{994}$. The ORF2 is predicted to start at the nt 990 which is the first base after the +1 PRF site and encodes a putative RdRp of 773 aa (Fig. 1a).

Pairwise comparison of the ORF1+2 fusion protein sequence of this virus with other amalgaviruses demonstrated the novel virus shared the identities from 34.9\% (Capsicum annuum 
amalgavirus 1, NC_040662) to 50.7\% (BBLV) (Supplementary Table 1), which is below the species demarcation criteria of $65-70 \%[13]$. A Neighbor-joining tree constructed using the fusion protein sequences placed this virus, BBLV and four other amalgaviruses in the same clade (Fig. 1b), there was no obvious host correlation among these amalgaviruses. Therefore, this virus should be considered a new species of the genus Amalgavirus, which is tentatively named Aconitum amalgavirus 1 (AcoAV-1).

\section{Occurrence on Chinese aconite plant and host range test of AcoAV-1}

To investigate AcoAV-1 infection in fields, a total of 17 symptomatic Chinese aconite plants including 5 plants used for HTS collected in Zhanyi were tested by RT-PCR using the AcoAV-1 specific primers, and 2 plants $(11.8 \%)$ were positive for the virus. Then 71 symptomatic Chinese aconite plant samples were collected from Wuding County, Yunnan Province for the detection of AcoAV-1, and 25 of them (35.2\%) were infected with the virus, indicating the virus is common in the crop. It is reported that amalgaviruses were transmitted very efficiently by seeds and associated with symptomless infections in their hosts $[14,15]$. Crude sap from AcoAV-1 positive sample was mechanically inoculated onto seedlings of cowpea (Vigna sinensis), pepper (Capsicum annuum), tomato (Solanum lycopersicum), Nicotiana glutinosa, N. tobacum, N. rustica and N. tabacum var. Xanthi nc, however, no viral symptom was observed and no AcoAV-1 was detected by RT-PCR from these inoculated plants at 20 days post inoculation. In this study, CMV and four other viruses were also identified in the HTS sample (data not show), so it is uncertain if AcoAV-1 are associated with the viral diseases on Chinese aconite plants. Characterization of the other viruses found in the HTS sample is currently on the way.

Acknowledgments: This study was funded by the Yunnan Academician Expert Workstation (202005AF150040).

\section{Compliance with ethical standards:}

Conflict of interest: The author declares no competing interests.

Ethical approval: This article does not contain any studies with human participants or animals performed by any of the authors. 


\section{References}

1. Ma Y, Yang YX, Shu XY, Huang J, Hou DB (2016) Aconitum carmichaelii Debeaux, cultivated as a medicinal plant in western China. Genet Resour Crop Evol 63(5):919-924.

2. Nyirimigabo E, Xu Y, Li Y, Wang Y, Agyemang K, Zhang Y (2015) A review on phytochemistry, pharmacology and toxicology studies of Aconitum. J Pharm Pharmacol 67(1):1-19.

3. Cohen J, Zeidan M, Rosner A, Gera A (2000) Biological and molecular characterization of a new carlavirus isolated from an Aconitum sp. Phytopathology 90(4):340-344

4. Fumiyoshi F, Shin-ichi F, Kouichi S, Masahide I (2008) Cucumber mosaic virus isolated from Aconitum spp. in Japan. J Gen Plant Pathol 74(1):88-905.

5. Wang R, Chen BW, Li Y, Cao MJ, Ding WL (2021) Complete nucleotide sequence of a new carlavirus infecting Aconitum carmichaelii in China. Arch Virol 166(5):1513-1515

6. Liu WX, Chen JS (2009) A double-stranded RNA as the genome of a potential virus infecting Vicia faba. Virus Genes 39(1):126-131

7. Dong YH, Lei XH, Li YL, Hussain MD, Zhou Y, Zhu XP, Zhou Tao (2019) Alarming the damage of seed-borne Southern tomato virus to tomato industry in China. Plant Protection 45(3): 254-256. (article in Chinese)

8. Dongbin P, Chul JG, Hyein K, Yoonsoo H (2018) Identification of two novel amalgaviruses in the common eelgrass (Zostera marina) and in Silico analysis of the amalgavirus +1 programmed ribosomal frameshifting sites. Plant Pathol J 34(2): 150-156

9. Nibert ML, Pyle JD, Firth AE (2016) A +1 ribosomal frameshifting motif prevalent among plant amalgaviruses. Virology 498:201-208

10. Krupovic M, Dolja VV, Koonin EV (2015) Plant viruses of the Amalgaviridae family evolved via recombination between viruses with double-stranded and negative-strand RNA genomes. Biology Direct 10:12.

11. Sabanadzovic S, Valverde RA, Brown JK, Martin RR, Tzanetakis IE (2009) Southern tomato virus: the link between the families Totiviridae and Partitiviridae. Virus Res 140(1-2):130-137

12. Lan PX, He P, Zhang YK, Zhang S, Zhang ZB, Chen XJ, Tan ST, Luo HM, Cao MJ, Li F(2019) Molecular characterization of a novel potyvirus infecting noni. Arch Virol 164(12):3099-3102

13. Depierreux D, Vong M, Nibert ML (2016) Nucleotide sequence of Zygosaccharomyces bailii virus Z: evidence for +1 programmed ribosomal frameshifting and for assignment to family 
Amalgaviridae. Virus Res 271: 115-124

14. Martin RR, Zhou J, Tzanetakis IE (2010) Blueberry latent virus: an amalgam of the Partitiviridae and Totiviridae. Virus Res 155(1):175-180

15. Park D, Hahn Y (2017) Genome sequences of Spinach deltapartitivirus 1, Spinach amalgavirus 1, and Spinach latent Virus identified in spinach transcriptome. J. Microbiol. Biotechnol 27(7):1324-1330 


\section{Figures}

a

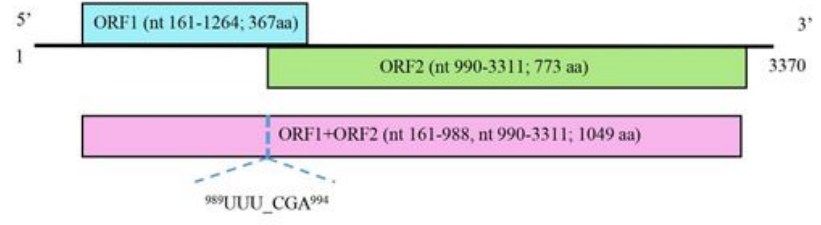

b

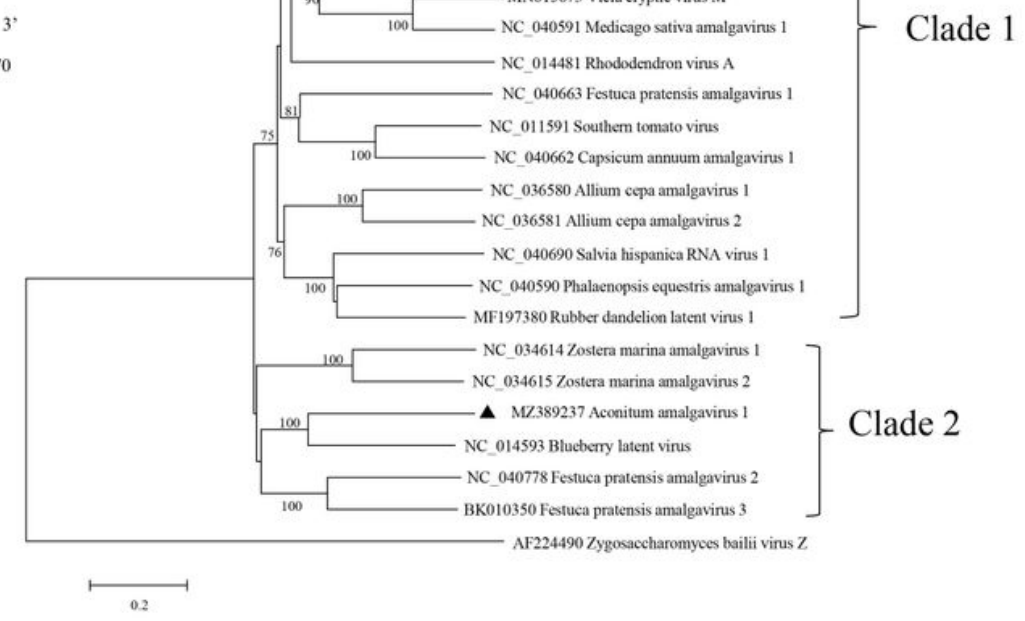

\section{Figure 1}

Characterization of a novel amalgavirus, Aconitum amalgavirus 1 (AcoAV-1), isolated from Chinese aconite plants in China. a Genomic organization of AcoAV-1 and the predicted +1 programmed ribosomal frameshifting(PRF) motif. b Neighbor-joining tree based on the theORF1+2 fusion protein amino acid sequences of AcoAV-1 and other related plant amalgaviruses. The fungus-infecting virus Zygosaccharomyces bailii virus Z (ZbV-Z) in the genus Zybavirus of the family Amalgaviridae was used as an out-group. The percentage of replicate trees in which the associated taxa clustered together in the bootstrap test (1000 replicates) are shown next to the branches. The scale bar representing a genetic distance of 0.2 .Solid triangle indicated the AcoAV-1 characterized in this study.

\section{Supplementary Files}

This is a list of supplementary files associated with this preprint. Click to download.

- table.pdf

- MZ389237AcoAV1.txt

- Supplementarytable.pdf 